\title{
Una mirada permanente al quehacer de la sociedad
}

\author{
A permanent look at the task the society
}

Julio César Orozco Alvarado ${ }^{1}$

Adolfo Alejandro Díaz Pérez ${ }^{2}$

\section{Resumen}

Una mirada permanente al quehacer de la sociedad es un ensayo de reflexión que como estudiantes, docentes e investigadores nos sitúan en el quehacer permanente del especialista de las ciencias sociales. Todo inicia a partir de la definición de nuestro objeto de estudio, siguiendo con el reconocimiento de las especializaciones de las disciplinas sociales, en donde nos establecemos para hacer un recorrido en sus campos de estudios y problemáticas sociales, a las cuales llamaremos anomia social. Finalmente, asumimos un conjunto de retos y desafíos que nos comprometen a ser y actuar como agentes del cambio social, desde nuestros respectivos micros-contextos hasta la sociedad en su conjunto, a través de la investigación y la promotoría social como método de intervención social.

Palabras clave: Ciencias Sociales, Investigación social, Promotoría social, Sociedad.

\section{Abstract}

A permanent look at the task of society is an essay of reflection that as students, teachers and researchers places us in the permanent task of the specialist in the social sciences. Everything starts from the definition of our object of study, continuing with the recognition of the specializations of social disciplines, where we set out to make a journey in their fields of studies and social problems, which we call social anomie. Finally, we assume a set of challenges and challenges that commit us to be and act as agents of social change, from our respective micros-contexts to society as a whole, through research and social promotion as a method of social intervention.

Key Words: Social Sciences, Research Social, Social Promoter, Society

\section{Introducción}

En el emundo de las Ciencias todas las disciplinas científicas tienen un objeto de estudio sobre el cual vuelcan sus métodos, técnicas e instrumentos de investigación para conocer esa área del conocimiento, interpretarla, explicarla, compararla y

1 Doctor en Educación e Intervención Social, Profesor Investigador de la Universidad Nacional Autónoma de Nicaragua-Managua (UNAN-MANAGUA). E-mail: jorozcoa@hotmail.com ORCID: https://orcid.org/oooo-0oo3-4819-0598

2 Licenciado en Ciencias de la Educación con Mención en Ciencias Sociales, Profesor de la Universidad Nacional Autónoma de Nicaragua-Managua (UNAN-MANAGUA). E-mail: adolfoalejandro73@yahoo.com ORCID: https://orcid.org/oooo-0002-4295-4094

Recibido: 01/11/2017 Aprobado: 01/01/2018

Orozco-Alvarado, J., \& Díaz-Pérez, A. (2018). Una mirada permanente al quehacer de la sociedad. Revista Electrónica de Conocimientos, 


\section{REFLEXIONES EDUCATIVAS}

fundamentar categóricamente y con todo el aplomo de un cientista social, porqué es importante y necesario su estudio. Y es a través de este fenómeno científico que surgen las especializaciones de las ciencias, en donde sus respectivos campos de estudios cada vez parecen ensanchar sus aristas, al abrir espacios de investigación a las nuevas problemáticas y tendencias que en la modernidad van surgiendo.

Las Ciencias Sociales, concebidas como "aquellas disciplinas científicas que estudian las actividades que desarrollan los seres humanos" (Serra, 2008, p.52), no están exentas de este fenómeno, pues desde sus distintas áreas disciplinares y especializaciones temáticas como la "antropología, economía, psicología, sociología, ciencias políticas, geografía, arqueología, demografía, derecho, educación, geografía humana, comunicación social, historia, trabajo social" (Serra, 2008, p.52) están abriendo espacios de estudio a los acontecimientos ocurridos en la actualidad, a través de la realización de procesos investigativos documentales, experimentales, comparativos, interpretativos, investigación-acción participativa, y estudios predictivos, en el caso de la psicología social, desde sus diversas disciplinas, lo cual ha generado un cúmulo de información concerniente a las relaciones que el ser humano establece entre sí y con su medio geográfico.

Por consiguiente, no cabe duda que las especializaciones de las ciencias sociales han aportado una gran cantidad de información sobre el ser humano y han abierto nuevos horizontes investigativos que no cesan de prolongarse, y esto es ventajoso para el especialista de las ciencias sociales, quien debe observar holísticamente el quehacer del ser humano en la sociedad para así comprender, interpretar y explicar la realidad social que subyace en cada contexto, sea este económico, político, social, cultural, educativo, etc.

Considerando estos aspectos, es importante destacar el rol que debe tener el especialista de las ciencias sociales ante la sociedad; a esto en el presente ensayo le hemos denominado sostener una mirada permanente en el quehacer de la sociedad.

\section{Reflexión educativa}

Situar la mirada en el quehacer de la sociedad es una acción disciplinada que todo cientista social debe poseer, pues es irrisorio que osemos explicar la realidad social si no estamos informados de lo que pasa en ella, además, es un imperativo profesional mirar permanentemente hacia la sociedad, ya que esta evoluciona constantemente, y debemos analizarla según las características que presentan en cada coyuntura, es decir, el científico social no puede rehusarse a los cambios de una sociedad que avanza de prisa y de manera vertiginosa.

De esto, un ejemplo sencillo lo encontramos en nuestro contexto nicaragüense, en donde los sucesos políticos contemporáneos (1979-1990; 1990-2006; 2007-actualidad) 
han traído consigo muchos cambios en la sociedad nicaragüense, desde transformaciones en el aparto político y socioeconómico, hasta en la renovación de los nombres oficiales de avenidas e institutos públicos del país. Por citar uno, el Aeropuerto de nuestro país, que fue fundado con el nombre Aeropuerto de las Mercedes en la década somocista (1968), en el periodo de la Revolución asumió el nombre de Aeropuerto Internacional Augusto C. Sandino (1979), posteriormente durante los gobiernos liberales ocupó el nombre de Aeropuerto Internacional de Managua (1990), y con el retorno del gobierno Sandinista en 2007 retomó el nombre de Aeropuerto Internacional Augusto C. Sandino.

Ante estos cambios sociales, políticos y económicos el cientista social no debe estar enajenado, y no debe juzgar a la sociedad con argumentos enraizados en el pasado, por el contrario, debe estar pendiente de los cambios acaecidos en los ámbitos macro y micro sociales, desde los aspectos más minúsculos como el mencionado, hasta los más estruendosos como el cambio de jurisdicción de la península de Crimea en Asia, que en 2014 pasó a soberanía rusa después de ser parte de Ucrania desde 1954 (Sánchez, 2016). A decir bien, sostener una mirada permanente en el quehacer de la sociedad implica asumir los cambios sociales como algo intrínseco a la sociedad.

Situarse en el quehacer de la sociedad es concebir el mundo como una red de comunicación o como un entramado de hechos sociales que trasciende las fronteras; un mundo en donde convergen las ideologías y en donde las ideas son premisas para la innovación y el emprendimiento, pero también son el origen de escenarios de confrontación y pugnas fratricidas, que inclusive, nos han llevado a poner en riesgo la existencia de la humanidad. Con respecto a esto $¿$ Por qué no le echamos un vistazo a las tensiones políticas entre Estados Unidos y Corea del Norte? La mirada del especialista de las ciencias sociales no debe estar alejada de esta realidad cercana y lejana a la vez, porque la globalización nos ha hecho interdependientes y conscientes que lo que afecta a los demás también nos puede afectar a nosotros pese a las latitudes que nos distancien.

Por las premisas antes planteadas, no cabe duda del por qué aludimos que el cientista social debe sostener una mirada permanente en las distintas facetas de desarrollo del ser humano en la sociedad, sea este político, económico, social, educativo, cultural, geográfico, histórico o filosófico, porque a fin de cuentas, todas ellas representan un área de desarrollo del ser humano y que como sociedad, reflejan el proceso históricosocial que la humanidad está gestando actualmente.

Pero, ¿Qué áreas del conocimiento deben ser objeto de observación permanente para el especialista de las ciencias sociales? A continuación se especificarán algunos de estos. 


\section{REFLEXIONES EDUCATIVAS}

En el caso de los acontecimientos de carácter históricos, el especialista en las ciencias sociales debe fijar su atención porque estos son una premisa imprescindible para comprender la realidad actual. La historia debe concebirse como una "interpretación del pasado" (Prats, 2001, p.43) que lejos de memorizarse y aprenderse mecánicamente, requiere de procesos cognitivos analíticos, interpretativos y críticos que nos acerquen a conocer y valorar la realidad en la que vivimos. Es decir, el cientista social establece vínculos con el pasado a fin de tener "una apreciación de cómo los problemas humanos han cambiado a través del pasado" (Pluckrose, 1993, p.17), a fin de trascender la visión tradicional que define la historia como aquella disciplina que únicamente "se ocupa del estudio del pasado y los acontecimientos que lo conforman" (Piña y Chávez, 2004, p.37).

Para contextualizar lo anterior, hagámonos la siguiente, ¿Podemos explicar la complejidad de las relaciones diplomáticas entre Nicaragua y Estados Unidos sin trascender a explorar los conflictos políticos, comerciales y geográficos que definieron las relaciones diplomáticas entre ambos países en el pasado? De igual manera, es impreciso hacer juicio a priori entre las tensas relaciones políticas que en la actualidad caracterizan a Venezuela y Estados Unidos, sino tomamos como punto de partida las políticas norteamericanas hacia América Latina en el siglo pasado. No obstante, el científico social debe sostener una mirada crítica y analítica a los acontecimientos históricos que anteceden a la sociedad que estudia.

La mirada del especialista en las ciencias sociales también debe estar puesta sobre los ámbitos económicos, los cuales fluctúan constantemente generando consigo abruptos cambios de políticas públicas, políticas económicas, inclusive, cambios de regímenes políticos. Muestra de ello fueron las reformar políticas, sociales y económicas que enmendaron los países satélites del socialismo soviético como Nicaragua, Ucrania y Polonia, cuando sucumbió el socialismo en la ex Unión de Repúblicas Socialistas Soviéticas (URSS), también conocida como Unión Soviética, en la actual Rusia.

En este sentido, dar seguimiento a las actividades económicas es prioritario para el cientista social que está enfocado en comprender el comportamiento de la sociedad. La economía, según Piña y Chávez (2004), es una disciplina social ambivalente por su tendencia cualitativa y cuantitativa, ya que se ocupa de realizar estudios acerca de las formas de producción, distribución y consumo de los bienes materiales que elaboran los seres humanos, además, estudia las formas de comportamientos de los mercados y su relación con el desarrollo de las sociedades.

Es por ello que afirmamos que el cientista social debe estar pendiente de las incidencias de las actividades económicas en la sociedad, tanto a nivel nacional como a nivel internacional, porque existe una estrecha relación entre los hechos que suscitan en el campo internacional con las actividades económicas nacionales. Ejemplo de ello es el caso de la explosión y hundimiento de la Deepwater Horizon [horizonte de aguas 
profundas] ocurrida el 20 de abril de 2010, la cual era una plataforma que perforaba pozos de petróleo, muriendo 11 personas, posteriormente se hundió, derramando unos 4,9 millones de barriles de petróleo a las aguas del Golfo de México, contaminando y matando la flora y la fauna marina.

Por otra parte, el científico social desde la sociedad en la que habita debe focalizar su mirada en las relaciones sociales que el ser humano establece entre sí, a esta área del conocimiento le denominamos psicología social. Según Piña y Chávez (2004), esta disciplina social "tiene como objeto de estudio a la persona. Estudia la naturaleza social del ser humano, y la manera que este se desarrolla. El desarrollo del ser humano se posibilita por el proceso de socialización" (p.35). Esta definición se complementada con la de Morris y Maisto (2009), quienes afirman que la psicología social estudia "las formas en que los pensamientos, sentimientos y conductas de un individuo reciben influencia de la conducta (...) de otras persona" (p.589).

Pero, en nuestro contexto, como cientistas sociales, ¿a qué debemos prestar atención según la psicología social? Aplicando los principios de la física cuántica que establece que una pregunta puede ser respondida con otra pregunta, podemos decir que, ¿Acaso el bullying que se da en las escuelas es un factor ajeno a la psicología social y al cientista social? ¿La inseguridad ciudadana producto de la delincuencia es un tópico ajeno a la psicología social y al cientista social? $\mathrm{O}$ ¿La poca articulación entre docentes, padres de familia y escuela no nos incumbe también a los científicos sociales? Con esto cabe destacar que la psicología social está presente en el quehacer científico del especialista de las ciencias sociales, por lo que este debe volcar su mirada hacia las problemáticas de orden psicosocial que están abatiendo a nuestras sociedades.

También debemos hacer notar que los aspectos socioculturales no están exentos del ámbito de estudio del especialista de las ciencias sociales. Por una parte, concebimos que la sociología "se ocupa de las relaciones sociales y de la organización estructural de la sociedad" (Rossetti, 2002, p.17), así mismo, cabe aclarar que la sociología "no estudia a individuos aislados, sino a comunidades y grupos, a individuos participantes en comunidades" (Piña y Chávez, 2004, p.22), esto comprende fijar la mirada permanente en aquellos indicadores sociales que inciden en el desarrollo de nuestra sociedad, por ejemplo la relación entre pobreza e inseguridad ciudadana; los aportes de la sociedad para el fortalecimiento de los valores colectivos; los aportes de la sociedad a la mejora de la calidad de los aprendizajes; o bien, la vinculación entre universidad y sociedad.

Asimismo, desde la perspectiva de la antropología el científico social se ocupa del componente cultural presente en nuestras sociedades. Rossetti (2002) fundamenta que la antropología se enfoca en las "diferentes formas de expresión cultural del hombre" (p.17). De acuerdo con esta perspectiva, es pertinente que desde las ciencias sociales le otorguemos un valor considerable a las expresiones culturales que nos caracterizan como sociedad, país y región, nos referimos a expresiones culturales como 
comidas, bebidas, juegos, costumbres, creencias etc., las cuales el científico social no puede eludir como objeto de estudio. Asimismo, este profesional debe hacer análisis permanente acerca de las similitudes y diferencias en cuanto a algunas costumbres que compartimos los habitantes de Latinoamérica y Centroamérica.

En vista de lo expuesto anteriormente, no cabe duda que el ámbito de estudio del especialista de las ciencias sociales es amplio y diverso, pues consiste en analizar las acciones del ser humano desde sus ámbitos políticos, económicos, sociales, geográficos etc. No obstante, también queremos exponer que el científico social al sostener una mirada permanente en el quehacer de la sociedad, también asume compromisos con la misma sociedad: es un promotor social y un investigador social.

El profesional de las ciencias sociales ancla su mirada en el quehacer de la sociedad porque además de estudiarla y analizarla, es un ciudadano comprometido por la calidad de las relaciones humanas, con alta sensibilidad humana y sentido de pertenencia a la madre tierra, proclive a la defensa y protección del medio ambiente. Es un soñador que ambiciona la calidad de la educación, a estudiantes ávidos por aprender, maestros con enormes motivaciones y razones para enseñar de manera innovadora, y a funcionarios educativos comprometidos con la escuela y la sociedad, es decir, visiona a profesionales comprometidos con la sociedad. En palabras de Serra (2008) "en las carreras de CC.SS 3 nos encontramos con una joven generación de estudiantes que, en su mayoría, posee una sensibilidad humana, vocación profesional e interés de contribuir al desarrollo del país" (p.57).

En cuanto al componente de investigación, este es un reto para el profesional de las ciencias sociales, pues ¿Qué sentido tiene sostener una mirada permanente en la sociedad si no desarrollamos procesos investigativos en la sociedad que observamos? No perdamos de vista que nuestra esencia como investigadores sociales es brindar un aporte a la sociedad, y esto lo lograremos a través de la investigación.

Nuestra sociedad, como decíamos anteriormente, tiene muchas dolencias, y en palabras de Mercado (2005), se podría decir que "al igual que el ser humano, la sociedad también sufre, con enfermedades que padece” (p.6o), a esto le denominamos anomia social. La anomia social es el conjunto de actividades disfuncionales y antisociales que se desarrollan en una determinada sociedad, en otras palabras, es un estado de irregularidad o alteración de las conductas normales practicadas en una sociedad. Por lo general estas alteraciones sociales son generadas por la incapacidad que tienen las estructuras sociales y políticas para proveer lo necesario a los individuos de la sociedad para que estos establezcan relaciones sociales basadas en la convivencia armoniosa (Mercado, 2005).

3 Significa Ciencias Sociales 
En este sentido, no cabe duda que nuestras comunidades representan un enorme reto para la investigación social, ya que nos situamos en un contexto marcado profundamente por la violencia, delincuencia, inseguridad social, inseguridad alimentaria, migración, pobreza, exclusión social, deterioro ambiental etc. Es ahí donde radica el desafío que tiene el científico social, ser un investigador en el micro contexto en donde este se desarrolla, a fin de generar espacios de cambios y transformación que conduzcan a la construcción de realidades sociales más justas y sostenibles.

Y es aquí en donde el componente de promotoría social se presenta como una inmanente cualidad que caracteriza al cientista social, porque sostener una mirada permanente en la sociedad, lo que implica promover el desarrollo sostenible de nuestras comunidades, es decir, no solo se trata de conocer e interpretar la realidad que se observa, sino también diseñar y ejecutar planes de intervención social que generen sostenibilidad y condiciones más justas para la sociedad y su entorno, es decir, si hay problemas educativos, ¿Por qué no crear una plan de intervención para elevar la calidad educativa?, si hay necesidades comunales ¿Por qué no ejecutar proyectos de desarrollo social?, no obstante, el científico social no es un jurista que cuestiona la realidad únicamente, no es un promotor de ideas solamente, el compromiso social es trascender, pues nos hace posicionarnos de roles activos que nos conducen de las ideas a las acciones, y de la investigación a la acción con el propósito de incidir en las problemáticas que adolece nuestra sociedad.

Las ciencias sociales deben guiar a los hombres en el largo y difícil camino histórico que se les presenta, para conformar una sociedad más justa y estable. Una sociedad cuyo sentido, objetivo y preocupación básica sea servir y educar al ser humano, logrando una convivencia donde seamos capaces de eliminar la miseria, la locura de la guerra, la violencia sin sentido. Una sociedad eminentemente humana, parecería ser la tarea urgente que hoy en día las ciencias sociales están llamadas a realizar (Mercado, 2005, p.61).

En vista de lo expuesto, Cipullo (2011) llevó a cabo una investigación socioeducativa de la cual se extrajeron los siguientes roles del promotor social:

- Tiene una clara concepción de la problemática actual.

- Fomenta la participación y cooperación de la comunidad para la identificación y solución de las problemáticas.

- Fomenta la apropiación de comportamientos sociales propicios para el desarrollo comunal.

- Promueve las transformaciones sociales. 


\section{REFLEXIONES EDUCATIVAS}

En esto y en otros roles encontramos el sentido esencial del cientista social, que sin lugar a dudas, consiste en sostener una mirada permanente en la sociedad para su incidencia y transformación. En palabras de Mercado (2005) "las ciencias sociales constituyen la más poderosa perspectiva analítica para explicar y proponer soluciones a las graves crisis que como sociedades unidas en un concepto 'mundo', nos aquejan" (p.58).

\section{Conclusiones y perspectivas futuras}

Una mirada permanente en el quehacer de la sociedad es una acción inherente en la vida del especialista de las ciencias sociales. Un cientista social no puede estar desvinculado de la realidad social, pues nuestro objeto de estudio -la sociedad- es cambiante en el espacio y en el tiempo, y debemos analizarla de acuerdo a las particularidades que cada coyuntura nos va presentando.

Asimismo, ser profesionales de las ciencias sociales implica asumir enormes retos y desafíos: como investigadores y educadores, para conocer, analizar e interpretar la sociedad; y como promotores, para ser agentes activos y promover cambios y transformaciones sociales en nuestros micros contextos, ¡Es por eso que el profesional de las ciencias sociales debe sostener una mirada permanente en el quehacer de la sociedad!

\section{Agradecimiento}

Agradecemos a las autoridades de la Facultad de Educación e Idiomas de la Universidad Nacional Autónoma de Nicaragua, Managua (UNAN- Managua), por el apoyo brindado en cuanto a fondo de tiempo para la realización del presente estudio.

\section{Lista de referencia}

Cipullo, M. (2011, noviembre). El rol del docente como promotor social en el contexto de la integración escuela-comunidad en la escuela integral bolivariana "Francisco Miranda". Revista científica digital del centro de investigación y estudios gerenciales, (2):96-110. Recuperado el 25 de enero de 2018 de, http://www.grupocieg. org/archivos_revista/2-2-8\%20(96110)\%20Cipullo\%2oMartha\%2orcieg\%20 noviembre\%2011_articulo_id73.pdf

Mercado, A. (2005). Ciencias Sociales: Retos Y Tendencias Temáticas. Rev. Ciencias Sociales. (III-IV), 57-73. Recuperado el 20 de enero de 2018, de http://www. redalyc.org/pdf/153/15323589005.pdf

Morris, C., y Maisto, A. (2009). Psicología. México: PEARSON EDUCACIÓN 
Piña, J. y Chávez, M. (2004). Introducción a las Ciencias Sociales. México: Publicaciones culturales.

Pluckrose, H. (1993). Enseñanza y aprendizaje de la Historia. Madrid: Morata, S.L.

Prats, J. (2001). Enseñar historia: notas para una didáctica renovadora. Mérida: Junta de Extremadura.

Rossetti, J. (2002). Introducción a la Economía. México: Alfaomega.

Sánchez, P. (2016, junio). El conflicto en ucrania: el primer enfrentamiento serio de Rusia con occidente durante la etapa de la post-guerra fría. Foro Internacional, (2):470-502. Recuperado el 08 de enero de 2018 de, http://www.redalyc.org/ pdf/599/59945401007.pdf

Serra, L. (2008). Retos de las Ciencias Sociales en Nicaragua. Encuentro, (79):52-62. Recuperado el 17 de enero de 2018 de http://repositorio.uca.edu.ni/320/1/ Encuentro79articulo7.pdf 